\title{
Lesões orais com potencial de malignização: análise clínica e morfológica de 205 casos
}

\section{Potentially malignant oral lesions: clinical and morphological analysis of 205 cases}

Éricka Janine Dantas da Silveira'; Maria Fernanda Fernandes Lopes²; Leonardo Miguel Madeira Silva²; Betania Fachetti Ribeiro; Kenio Costa Lima ${ }^{4}$; Lélia Maria Guedes Queiroz ${ }^{5}$

$\quad$ unitermos
Lesões orais
potencialmente malignas
Leucoplasia
Eritroplasia
Queilite actínica

\section{resumo}

Objetivos: O objetivo desta pesquisa foi realizar uma análise clínica e morfológica em lesões orais com potencial de malignização. Material e método: Os dados clínicos e morfológicos referentes a tais lesões foram obtidos a partir das fichas clínicas e dos laudos histopatológicos, respectivamente. Resultados: Dos 205 casos analisados, a lesão mais prevalente foi a leucoplasia (70,7\%), seguida da queilite actínica $(16,1 \%)$, eritroplasia $(9,8 \%)$ e eritroleucoplasia $(3,4 \%)$. Verificou-se uma associação significativa entre o sexo e as lesões $(p<0,001)$, sendo o sexo feminino mais prevalente nas leucoplasias e eritroplasias. A raça branca foi mais acometida em todas as lesões. Quanto à localização anatômica, o rebordo alveolar prevaleceu na leucoplasia (21\%), na eritroplasia e eritroleucoplasia, o palato foi mais acometido com $47,4 \%$ e $57,1 \%$, respectivamente. Com relação à idade, não houve diferença significativa entre as lesões $(p>0,05)$, sendo mais frequentes nas quinta, sexta e sétima décadas de vida. Observou-se que, na maioria dos casos de eritroplasias e eritroleucoplasias, os diagnósticos clínicos foram incompatíveis com o morfológico. Verificou-se associação significativa entre transformação maligna e lesões $(p<0,001)$, pois a eritroplasia apresentou uma taxa de malignização de $20 \%$, enquanto na leucoplasia o índice foi de 2,1\%. Conclusão: A prevalência das lesões orais com potencial de malignização foi semelhante ao encontrado na literatura, mas com algumas diferenças em relação ao perfil epidemiológico das mesmas, ressaltando-se que estas entidades merecem uma atenção especial por parte do clínico, especialmente as eritroplasias, que apresentaram um elevado percentual de malignização e o maior índice de equívocos no diagnóstico clínico.

abstract

Objective: The objective of this study was to perform a clinical and morphological analysis of potentially malignant oral lesions. Material and method: The clinical and morphological data related to these lesions were obtained from clinical and histopathological records, respectively. Results: Out of 205 cases, the most prevalent lesion was oral leukoplakia (70.7\%), followed by actinic cheilitis (16.1\%), erythroplakia (9.8\%) and erythroleukoplakia (3.4\%). There was a significant association between the lesions and gender $(p<0.001)$. The female gender was the most affected in leukoplakias and erythroplakias. Caucasians were mostly affected in all conditions. As far as anatomical location is concerned, the alveolar ridge was the most prevalent site in oral leukoplakias (21\%); the palate was the most affected in erythroplakia (47.4\%) and erythroleukoplakia (57.1\%). There was no significant difference among the lesions as to age ( $p>0.05)$, although they were more frequent in the 5th, 6th and 7th decades of life. In most cases of erythroplakias and erythroleukoplakias, it was observed that clinical and morphological diagnoses were incompatible. There was a significant association between malignant transformation and lesions $(\mathrm{p}<0.001)$ as the erythroplakia presented a malignization rate of $20 \%$, whereas in leukoplakias the rate was $2.1 \%$. Conclusion: The prevalence of potentially malignant oral lesions was similar to other findings in the medical literature, but with some differences in their epidemiological profile. It is important to highlight that these entities require further medical investigation, mainly erythroplakias, which showed high risk of malignization and the highest rate of clinical misdiagnosis.

\section{key words}

Potentially malignant oral lesions

Leukoplakia

Erythroplakia

Actinic cheilitis

1. Doutora em Patologia Oral pela Universidade Federal do Rio Crande do Norte (UFRN) e professora da disciplina de Patologia Oral da UFRN.

2. Graduandos em Odontologia pela UFRN e bolsista de iniciação científica da base de pesquisa em Patologia Oral da UFRN.

3. Mestre em Patologia Oral pela UFRN.

4. Doutor em Ciências (Microbiologia Médica) pela Universidade Federal do Rio de Janeiro (UFRI) e professor adjunto da UFRN.

5. Doutora em Patologia Oral pela UFRN e professora da disciplina de Patologia Oral da UFRN.

Programa de Pós-Graduação em Patologia Oral da UFRN. 


\section{Introdução}

As lesões pré-malignas ou lesões precursoras do câncer são alterações teciduais que podem assumir o caráter de tumor maligno, a qualquer tempo, mas, por outro lado, podem permanecer estáveis por um considerável período de tempo ${ }^{(7)}$. Em 2005, a Organização Mundial da Saúde (OMS) modificou a terminologia das lesões e condições orais pré-malignas e as denominou lesões com potencial de malignização ${ }^{(14)}$, citando-se nessa classificação, como lesões potencialmente malignas, leucoplasia, leucoplasia verrucosa proliferativa, eritroplasia, queilite actínica, fibrose submucosa, líquen plano e atrofia por deficiência de ferro.

Leucoplasia é um termo clínico utilizado para denominar uma placa predominantemente branca da mucosa oral, não removível à raspagem, que não pode ser classificada clinicamente em qualquer outra entidade. Sua superfície pode apresentar-se lisa, rugosa ou verrucosa, sendo a lesão precursora mais frequente da boca ${ }^{(3)}$. Acomete principalmente a mucosa jugal e as comissuras labiais, seguidas por mucosa alveolar, língua, lábio, palato duro, palato mole, assoalho de boca e gengiva ${ }^{(7)}$. A taxa de transformação maligna de leucoplasia varia de $0 \%$ a $20 \%$, sendo em média de $5 \%$ (13).

A eritroplasia é definida como uma placa ou mancha vermelha que não pode ser classificada clinicamente como qualquer outra entidade. Essa pode associar-se a uma leucoplasia adjacente, sendo denominada, nesse caso, eritroleucoplasia $^{(7)}$. Apesar de ser menos comum que a leucoplasia, a eritroplasia apresenta maior potencial para transformação maligna ( $14 \%$ a $50 \%$ ), pois em cerca de $90 \%$ dos casos é uma displasia moderada ou severa ${ }^{(14)}$.

A queilite actínica é uma condição de natureza inflamatória e potencialmente maligna, que acomete o lábio inferior, causada pela exposição prolongada e crônica aos raios solares. As lesões são geralmente assintomáticas, podendo ser brancas, vermelhas, brancas com áreas vermelhas e também ulceradas. É relatado que a taxa de transformação maligna varia de $10 \%$ a $20 \%{ }^{(6)}$.

Em virtude da escassez de trabalhos publicados no tocante ao perfil de pessoas acometidas pelas lesões com potencial de malignização da mucosa oral, o objetivo desse trabalho consiste na realização de uma análise clínica e morfológica nessas lesões em um período de 37 anos, especificamente em leucoplasias, eritroplasias e queilites actínicas, por serem mais comuns na região do estudo e por não haver controvérsias no tocante ao seu potencial de malignização. Adicionalmente, propõe-se verificar a relação do diagnóstico clínico com as alterações morfológicas encontradas nas referidas lesões, haja vista em muitas situações o clínico sugerir uma hipótese diagnóstica de lesão potencialmente maligna e histologicamente a mesma não representar esse tipo de alteração.

\section{Material e método}

O presente estudo foi baseado nos casos de lesões com potencial de malignização arquivados no Serviço de Anatomia Patológica do Departamento de Odontologia da Universaidade Federal do Rio Grande do Norte (UFRN), no período de janeiro de 1970 a janeiro de 2007.

Os dados clínicos referentes a tais condições foram obtidos a partir das fichas clínicas arquivadas no referido serviço e do diagnóstico histopatológico obtido a partir dos laudos histopatológicos dos referidos casos.

Uma ficha previamente elaborada foi utilizada para coletar informações sobre sexo, idade e raça, assim como localização anatômica, diagnóstico clínico e histopatológico. Após obtenção dos dados, os diagnósticos clínicos e histológicos foram agrupados em duas categorias: compatíveis, quando havia compatibilidade entre o diagnóstico clínico e o histopatológico; e não-compatível, quando os achados morfológicos não eram condizentes com os aspectos clínicos, ou seja, quando o diagnóstico histopatológico não era displasia epitelial, carcinoma in situ ou invasivo, o mesmo era considerado não-compatível com o clínico.

Os dados coletados foram utilizados para a construção de um banco de dados em uma planilha do Excel, os quais foram posteriormente transferidos para o software SPSS 13.0. O teste do qui-quadrado foi utilizado para identificar associação entre as variáveis sexo, raça e diagnóstico histopatológico compatível com as lesões pré-malignas. Em seguida, a análise de variância (ANOVA) foi utilizada para verificar a diferença entre a variável independente idade e os tipos de lesões pré-malignas.

O presente trabalho foi aprovado pelo Comitê de Ética em Pesquisa (CEP) da Universidade Federal do Rio Grande do Norte (UFRN), sob o Parecer 179/2007.

\section{Resultados}

De acordo com os critérios mencionados, de um total de 7.725 casos do no Serviço de Anatomia Patológica 
do Departamento de Odontologia da UFRN, 205 (2,6\%) tinham diagnóstico clínico de lesões com potencial de malignização. Dessas, a lesão mais prevalente foi a leucoplasia, com 70,7\% (145 casos), seguida da queilite actínica, com 16,1\% (33 casos), eritroplasia, com 9,8\% (20 casos) e eritroleucoplasia, com 3,4\% (sete casos).

Os dados em relação ao sexo, raça, localização e idade encontram-se listados na Tabela 1.

Para verificar a associação entre as variáveis sexo, raça e idade e as lesões com potencial de malignização por meio do teste qui-quadrado, uniram-se os casos de eritroleucoplasia aos de eritroplasia, verificando-se associação significativa entre o sexo e as lesões $(p<0,001)$. Houve predileção da leucoplasia e eritroplasia pelo sexo feminino e da queilite actínica, pelo masculino. Na eritroleucoplasia, verificou-se uma equivalência em ambos os sexos.

No tocante à idade, não houve diferença significativa entre essa variável e as lesões pré-malignas $(p=0,169)$, sendo a média de idade maior para os pacientes portadores de eritroplasias (60,63 anos).

Com relação ao diagnóstico histopatológico, alguns não estavam de acordo com o diagnóstico clínico, e foram, portanto, incluídos na categoria não-compatível, como pode ser verificado na Tabela 2.

Distribuição das variáveis sexo, raça e localização anatômica entre as lesões pré-malignas, e média, Tabela 1 DP e intervalo de confiança da idade dos indivíduos portadores dessas lesões (Natal/RN, 2007)

\begin{tabular}{|c|c|c|c|c|}
\hline \multirow{2}{*}{ Variável } & \multicolumn{4}{|c|}{ Lesão } \\
\hline & Leucoplasia & Queilite actínica & Eritroplasia & Eritroleucoplasia \\
\hline \multicolumn{5}{|l|}{ Sexo } \\
\hline Masculino & $54(37,2 \%)$ & $28(84,8 \%)$ & $2(10 \%)$ & $3(42,9 \%)$ \\
\hline Feminino & $91(62,8 \%)$ & $5(15,2 \%)$ & $18(90 \%)$ & $4(57,1 \%)$ \\
\hline \multicolumn{5}{|l|}{ Raça* } \\
\hline Branca & $84(67,7 \%)$ & $21(91,3 \%)$ & $6(54,5 \%)$ & $3(75 \%)$ \\
\hline Não Branca & $40(32,3 \%)$ & $2(8,7 \%)$ & $5(45,5 \%)$ & $1(25 \%)$ \\
\hline \multicolumn{5}{|l|}{ Localização* } \\
\hline $\begin{array}{l}\text { Rebordo } \\
\text { alveolar }\end{array}$ & 30 (21\%) & - & $3(15,8 \%)$ & - \\
\hline Mucosa jugal & $26(18,2 \%)$ & - & $3(15,8 \%)$ & - \\
\hline Palato & $25(17,5 \%)$ & - & $9(47,4 \%)$ & $4(57,1 \%)$ \\
\hline Gengiva & $15(10,5 \%)$ & - & - & - \\
\hline Língua & $13(9,1 \%)$ & - & - & - \\
\hline Lábio inferior & $13(9,1 \%)$ & 33 (100\%) & - & $1(14,3 \%)$ \\
\hline $\begin{array}{l}\text { Região } \\
\text { retromolar }\end{array}$ & $9(6,3 \%)$ & - & $1(5,3 \%)$ & $1(14,3 \%)$ \\
\hline $\begin{array}{l}\text { Assoalho } \\
\text { bucal }\end{array}$ & $1(0,7 \%)$ & - & & - \\
\hline $\begin{array}{l}\text { Sulco } \\
\text { vestibular }\end{array}$ & $1(0,7 \%)$ & - & $1(5,3 \%)$ & - \\
\hline $\begin{array}{l}\text { Múltiplas } \\
\text { localizações }\end{array}$ & $10(7 \%)$ & - & $2(10,5 \%)$ & $1(14,3 \%)$ \\
\hline \multicolumn{5}{|l|}{ Idade* } \\
\hline$x \pm D P$ & $53,99 \pm 15,1$ & $51,64 \pm 14,2$ & $60,63 \pm 15,1$ & $60,29 \pm 14,5$ \\
\hline IC (95\%) & $51,47-56,52$ & $46,61-56,66$ & $52,56-68,69$ & $46,83-73,74$ \\
\hline
\end{tabular}

*Essas variáveis não se encontravam preenchidas em algumas fichas clínicas.

x: média de idade (em anos); DP: desvio padrão; IC: intervalo de confiança. 


\section{Tabela 2}

Números absolutos e percentual válido dos diagnósticos histopatológicos compatíveis e não-compatíveis para cada lesão pré-maligna (Natal/RN, 2007)

Lesões pré-malignas

Diagnóstico

histopatológico*

Compatível

Não-compatível
Leucoplasia

$105(73,4 \%)$

$38(26,6 \%)$
Queilite actínica

$22(66,7 \%)$

$11(33,3 \%)$
Eritroplasia

$7(35 \%)$

$13(65 \%)$
Eritroleucoplasia

$5(71,4 \%)$

$2(28,6 \%)$

*Dois diagnósticos histopatológicos encontravam-se ausentes nas fichas clínicas.

Houve associação significativa entre as categorias compatível e não-compatível e as lesões $(p=0,011)$, podendo ser observado que a união dos casos de eritroleucoplasia e eritroplasia apresentou maior quantidade de diagnósticos histopatológicos não-compatíveis, enquanto nas leucoplasias e queilites actínicas o diagnóstico compatível prevaleceu.

Entre os diagnósticos não-compatíveis, os mais comuns foram líquen plano e hiperplasia fibrosa inflamatória, com respectivamente $3,4 \%$ e $2,5 \%$.

Quanto ao diagnóstico compatível das leucoplasias, a hiperceratose foi a mais prevalente $(46,2 \%)$, estando associada a displasias em 7,7\% e apenas displasias em 19,6\%. Nas queilites actínicas, 45,5\% dos casos apresentaram apenas elastose solar no tecido conjuntivo e atrofia epitelial, associando-se à displasia leve em $15,2 \%$, displasia moderada em $9,1 \%$ e hiperceratose em $9,1 \%$. Displasias de leve a severa foram encontradas isoladas em $21,1 \%$ dos casos.

No tocante às eritroplasias, 30\% eram displasias, sendo $20 \%$ displasia leve, $5 \%$ displasia moderada e 5\% displasia severa. Nas eritroleucoplasias, a hiperceratose foi a mais prevalente, com $57,1 \%$ dos casos, sendo também encontrados $14,3 \%$ de displasia epitelial leve. Em relação às displasias, o teste qui-quadrado não foi aplicável.

O teste qui-quadrado foi aplicado para verificar a associação entre transformação maligna e lesões pré-malignas, observando-se significância estatística $(p<0,001)$, pois a eritroplasia apresentou uma taxa de transformação maligna de $20 \%$, enquanto na leucoplasia essa taxa foi de apenas 2,1\%. A queilite actínica e a eritroleucoplasia apresentaram uma taxa de malignização de $12,1 \%$ e $14,28 \%$, respectivamente.

\section{Discussão}

A escassez de pesquisas acerca do perfil da população acometida pelas lesões com potencial de malignização da mucosa oral (leucoplasias, eritroplasias, eritroleucoplasias e queilites actínicas) e a diversidade de entidades patológicas que podem manifestar-se clinicamente como as lesões, motivou-nos a realizar este estudo.

Segundo relatos de Lee et al.(5), a leucoplasia é a lesão cancerizável mais frequente da cavidade bucal, o que concorda com os nossos achados, pois essa lesão correspondeu a $70,7 \%$ dos casos.

Dietrich et al..$^{(1)}$ e Lee et al. ${ }^{(5)}$ encontraram em seus estudos uma alta prevalência da leucoplasia em homens, o que vai de encontro aos resultados deste estudo, já que as mulheres foram mais acometidas $(62,8 \%)$. Nas pesquisas de Freitas et al. ${ }^{(2)}$, do total de 52 pacientes, $65 \%$ eram homens, mas $78 \%$ desse grupo eram fumantes, enquanto $82 \%$ dos pacientes não-fumantes eram mulheres. Apesar de essa informação não constar em nossas fichas clínicas, acreditamos que a maioria da nossa amostra é de nãofumantes, devido ao fato de haver maior incidência das lesões em mulheres.

Nas pesquisas de Lee et al.(5), consta que as localizações anatômicas mais frequentes das leucoplasias foram a mucosa jugal e a língua, o que discorda do presente estudo, visto que o rebordo alveolar foi o sítio anatômico mais acometido (21\%), seguido da mucosa jugal $(18,2 \%)$ e do palato $(17,5 \%)$.

Gabriel et $a l^{(3)}$ relataram uma maior prevalência da leucoplasia entre a quarta e a sexta décadas de vida. De acordo com Dietrich et al. ${ }^{(1)} \mathrm{e}$ Neville et al. ${ }^{(7)}$, essas lesões ocorrem predominantemente em pessoas com idade superior a 50 anos. Esse resultado está em consonância com os nossos achados, em que houve uma prevalência entre a quarta e a sétima décadas de vida, sendo a idade média dos pacientes acometidos pela leucoplasia de 53,99 anos.

Conforme Rodrigues et al.(11), o processo de diagnóstico da leucoplasia é um tanto complexo, não só pela diversidade de seus aspectos clínicos, mas também pela ausência de sintomatologia, sendo geralmente descoberta em exames de rotina e, por isso, torna-se necessário fazer 
um diagnóstico por exclusão de outras lesões que se apresentam como placas brancas na mucosa bucal.

Segundo esse mesmo grupo de autores, a leucoplasia possui um quadro histológico variado, mas duas características são consideradas importantes: a hiperceratose e a displasia epitelial em vários graus de severidade. Essas alterações são significativas, do ponto de vista evolutivo da lesão, por determinarem comportamentos biológicos diferentes e, consequentemente, diferente acompanhamento clínico.

Considerando apenas os diagnósticos histológicos que foram compatíveis com o diagnóstico clínico, a hiperceratose foi o mais prevalente, com $46,2 \%$. Entre os graus de displasia encontrados nos achados histopatológicos, a displasia epitelial leve foi a mais encontrada $(71,85 \%)$, achados esses semelhantes aos da pesquisa de Rodrigues et al. ${ }^{(11)}$. Apesar de o aspecto clínico das leucoplasias estar omitido em grande parte das nossas fichas clínicas, acreditamos, com base nas pesquisas desse grupo de autores, que a maioria dos nossos casos era de leucoplasias homogêneas, haja vista que os diagnósticos morfológicos denotaram em sua maior parte a presença apenas de hiperceratose e displasia epitelial leve.

Nas pesquisas de Gabriel et al. ${ }^{(3)}$, consta que a alta prevalência da leucoplasia oral requer atenção especial dos cirurgiões-dentistas que podem, ao exame físico, identificar áreas suspeitas e diagnosticá-las, pois, devido ao seu risco de transformação maligna, encontrado também no presente estudo, requer intervenção terapêutica adequada, a qual inclui identificação e remoção dos fatores de risco, bem como controle periódico do paciente, devido à possibilidade de recidivas.

De acordo com Neville et al. ${ }^{(7)}$, a eritroplasia oral é muito menos comum que a leucoplasia oral, concordando com nossos resultados, que mostraram uma prevalência de 9,8\% entre a população analisada, mais comum em homens, o que vai de encontro aos nossos achados, pois observou-se maior prevalência no sexo feminino (90\%).

Na presente pesquisa, o sítio mais acometido pela eritroplasia foi o palato $(47,4 \%)$, seguido pela mucosa jugal e rebordo alveolar, ambos com 15,8\% dos casos. Segundo Hashibe et al. ${ }^{(4)}$, a eritroplasia ocorre principalmente em pessoas de meia idade e em idosos, isso também pode ser verificado na nossa pesquisa, pois essa lesão prevaleceu nas sexta e sétima décadas de vida, com uma média de idade de 60,63 anos, sendo a maior média de idade das condições estudadas.
Histologicamente, 90\% das eritroplasias apresentam displasia epitelial grave, carcinoma in situ e carcinoma de células escamosas ${ }^{(7,8)}$. Nos nossos achados, das 20 lesões diagnosticadas clinicamente como eritroplasia, cinco apresentavam displasia e quatro eram carcinomas, sendo um carcinoma in situ e três, carcinomas de células escamosas. Portanto, apesar de ter sido menos comum que a leucoplasia, a eritroplasia apresentou neste estudo maior potencial de sofrer transformação maligna, concordando com os achados da literatura.

Em nosso estudo, preocupantes foram os resultados em relação à concordância do diagnóstico clínico com o morfológico para as eritroplasias orais, pois, em grande parte dos casos diagnosticados como eritroplasia, não havia concordância com os achados histopatológicos, devendo chamar atenção dos clínicos para maiores cuidados no estabelecimento das suspeitas clínicas e na eliminação de qualquer outro diagnóstico quando da emissão do diagnóstico clínico de eritroplasia.

A queilite actínica é uma condição patológica que afeta o lábio inferior, que se apresenta com um aspecto atrófico, pálido e frequentemente com fissuração ${ }^{(8)}$. Em relação a essa condição, no presente estudo, $84,8 \%$ dos pacientes eram do sexo masculino, o que concorda com estudos realizados por Markopoulous et al.(6), em que essa prevalência foi de $54 \%$.

Richard et al. ${ }^{(10)}$ relataram que a queilite é encontrada mais em idosos. Já Markopoulos et al. ${ }^{(6)}$ encontraram em seus estudos pessoas com uma idade média de 53,1 anos acometidas por essa condição, o que está de acordo com resultados desta pesquisa, em que a idade média dos pacientes foi de 51,64 anos, sendo a quinta década de vida predominante.

Encontrou-se uma associação significativa entre transformação maligna e lesões $(p<0,001)$, pois a eritroplasia apresentou uma taxa de malignização superior à da leucoplasia, $20 \%$ e $2,1 \%$, respectivamente, o que está de acordo com os estudos de Scheifele et al. ${ }^{(12)}$. Conforme relatos de Reichart et al.(9), a transformação maligna das eritroplasias orais varia de $14 \%$ a $50 \%$, concordando esse índice com os resultados do presente estudo, assim como ocorre com a taxa de malignização da queilite actínica $(12,1 \%)$.

Com a realização desta pesquisa, verificaram-se algumas semelhanças e diferenças com os poucos artigos publicados na literatura no tocante ao perfil epidemiológico das lesões orais com potencial de malignização, já que a leucoplasia foi a mais frequente na boca e com menor taxa de transformação maligna, discordando apenas no fato de que 
as mesmas, juntamente com as eritroplasias, em nossa amostra, foram mais prevalentes em mulheres. Ressalta-se ainda que o clínico deve denotar uma maior atenção aos aspectos clínicos das lesões vermelhas da mucosa bucal, visto que em grande parte de nossa amostra o diagnóstico clínico de eritroplasia não era compatível com o histológico de displasias, reforçando-se ainda um controle especial dos pacientes portadores dessa condição, em virtude do risco de transformação maligna das lesões. Atenção especial também deve ser conferida aos agricultores e às pessoas que trabalham expostas ao sol, visto que as queilites actínicas são comuns nesses grupos de pessoas e também exibe risco de transformação maligna considerável, conforme reforçado por nosso estudo.

\section{Referências}

1. DIETRICH, T.; REICHART, P.A.; SCHEIFELE, C. Clinical risk factors of oral leukoplakia in a representative sample of the US population. Oral Oncol, v. 40, n.2, p. 15863, 2004

2. FREITAS, M.D. et al. Clinicopathologic aspects of oral leukoplakia in smokers and nonsmokers. Oral Surg Oral Med Oral Pathol Oral Radiol Endod, v.102, n.2, p.199-203, 2006.

3. GABRIEL, J.G. et al. Considerações gerais e abordagem clínica da leucoplasia oral. Rev Bras Patol Oral, v. 3, n.4, p.187-94, 2004.

4. HASHIBE, M. et al. Chewing Tobacco, Alcohol, and the Risk of Erythroplakia. Cancer Epidemiol, Biomarkers Prev, v.9, p.639-45, 2000.

5. LEE, J.J. et al. Carcinoma and dysplasia in oral leucoplakias in Taiwan: Prevalence and risk factors. Oral Surg Oral Med Oral Pathol Oral Radiol Endod, v.101, n.4, p. 472-9, 2006

6. MARKOPOULOS, A.; ALBANIDOU-FARMAKI, E.; KAYAVIS, I. Actinic cheilitis: clinical and pathologic characteristics in 65 cases. Oral Dis, v.10, p.212-6, 2004.

7. NEVILLE, B. W. et al. Patologia Oral \& Maxilofacial. 2 ed. Rio de Janeiro, RJ: Guanabara Koogan, 2004.
8. REGEZI, J. Á.; SCIUBBA, J. J.; JORDAN, R. C. K. Patologia Bucal - Correlações Clinicopatológicas. 5 ed. Rio de Janeiro, RJ: Elsevier, 2008.

9. REICHART, P.A.; PHILIPSEN, H.P. Oral Erythroplakia - A review. Oral Oncol, v.41, p.551-61, 2004.

10. RICHARD, A.L.; WILDE, J.L.; GRABSKI, W.J. Comparison of Electrodessication with CO2 Laser for the Treatment of Actinic Cheilitis. Dermatol Surg, v.26, p.349, 2000.

11. RODRIGUES, T.L.C. et al. Leucoplasias bucais: relação clínico-histopatológica. Pesqui Odontol Bras, v.14, n.4, p. 357-61, 2000.

12. SCHEIFELE, C.; Reichart, P.A. Is there a natural limit of the transformation rate of oral leukoplakia? Oral Oncol, v.39, n.5, p.470-5, 2003.

13. SCHEPMAN, K.P. et al. Malignant transformation of oral leukoplakia: a follow-up study of a hospital-based population of 166 patients with oral leukoplakia from The Netherlands. Oral Oncol, v.34, n.4, p.270-5, 1998.

14. VAN DER WAAL, I. Potentially malignant disorders of the oral and oropharyngeal mucosa; terminology, classification and present concepts of management. Oral Oncol, v. 45, n. 4-5, p. 317-23, 2009. 